\title{
Effects of Divalent Ions on Vesicle-Vesicle Fusion Studied by a New Luminescence Assay for Fusion
}

\author{
Ronald W. Holz and Carol A. Stratford \\ Department of Pharmacology, University of Michigan Medical School, \\ Ann Arbor, Michigan 48109
}

Received 27 October 1978; revised 2 January 1979

Summary. A new assay has been developed for vesicle-vesicle fusion based upon the mixing of intravesicular contents of two sets of vesicles. Purified firefly luciferase and $\mathrm{MgCl}_{2}$ were incorporated into one set of vesicles (LV) and ATP into the other (AV). Vesicles were prepared from soybean phospholipids. The luminescence that resulted from hydrolysis of ATP by luciferase was measured to determine the extent of mixing of the intravesicular contents. In the absence of divalent ions, incubation of a mixture of $\mathrm{LV}$ and $\mathrm{AV}$ did not produce luminescence. However, if $\mathrm{Ca}^{++}$or other divalent ions were present at millimolar concentrations, luminescence occurred. The luminescence did not result from extravesicular reaction of vesicle contents that had leaked into the medium. Instead, luminescence resulted from the mixing of intravesicular spaces of AV and LV in fused vesicles. Optical density changes and negative stain electron microscopy indicated that $\mathrm{Ca}^{++}$induced extensive aggregation of vesicles. However, quantitation of the maximum possible luminescence indicates that only a small percentage (less than $1 \%$ ) of the vesicles actually fused in a fusion experiment.

Addition of EDTA to chelate $\mathrm{Ca}^{++}$after luminescence had been induced resulted in a two- to threefold increase in light emission which then rapidly decayed. These results suggest that the sudden removal of $\mathrm{Ca}^{++}$caused a transient increase in fusion after which subsequent fusion was inhibited. It was also found that the vesicles were relatively stable to hypotonic solutions.

Membrane fusion has been implicated in the control of various biological phenomena, including neurotransmission, endocytosis, exocytosis, fertilization, and cell fusion. In vitro studies of membrane fusion have utilized artificial lipid vesicles (Papahadjopoulos et al., 1974; Miller \& Racker, 1976), naturally occurring secretory vesicles (Lawson et al., 1977; Grazl \& Dahl, 1978), and cells (Ahkong et al., 1973; Martin \& MacDonald, 1976). Lawson et al. (1977) have demonstrated that exocytosis in rat peritoneal mast cells involves fusion between the lipid bilayer components of the membranes with displacement of membrane proteins from the site of fusion. Thus, studies of membrane fusion using artificial vesicles may illuminate aspects of lipid-lipid interactions that are important in the biological process. 
Fusion studies of artificial lipid vesicles have yielded information concerning the effects of transmembrane osmotic gradient (Miller et al., 1976), lipid composition and fluidity (Papahadjopoulos et al., 1974; Miller \& Racker, 1976), and pH (Breisblatt \& Ohki, 1976) on membrane fusion. In such studies, a critical problem has been development of a suitable and sensitive assay to determine whether fusion has occurred. Evidence for vesicular fusion has been provided by such techniques as transfer of radiolabelled phospholipids (Papahadjopoulos et al., 1974), calorimetry (Papahadjopoulos et al., 1974), and transfer of specific membrane proteins (Miller \& Racker, 1976). However, direct proof of vesicle fusion requires mixing of intravesicular contents. Such proof has been provided by a study in which the calcium-sensitive dye, arzenazo III was mixed with EDTA through vesicle fusion to cause a spectral light shift (Dunham et al., 1977). More recently, Ingolia and Koshland (1978) have reported a vesicular fusion assay which utilizes the reaction of firefly extract with ATP to produce luminescence. We have developed a similar assay for vesicle-vesicle fusion using purified firefly luciferase, the cofactor luciferin, and ATP.

The luciferase enzyme reaction of light production involves a two-step process (DeLuca \& McElroy, 1974):

1) Luciferase +luciferin $+\operatorname{ATP} \stackrel{\mathrm{Mg}^{++}}{\longrightarrow}$

luciferase-luciferin $-\mathrm{AMP}+\mathrm{PP}_{i}$

2) Luciferase-luciferin-AMP $+\mathrm{O}_{2}$

$$
\text { oxyluciferin-luciferase }+\mathrm{CO}_{2}+\mathrm{AMP}+\text { light. }
$$

Whereas use of the firefly extract has been reported to be sensitive to picomolar quantities of ATP (Stanley \& Williams, 1969), use of purified firefly luciferase and luciferin allows even greater sensitivity (Kimmich, Randles \& Brand, 1975). Thus, the purified system potentially provides a more sensitive assay for vesicular fusion.

In the present study we have incorporated purified firefly luciferase and $\mathrm{Mg}^{++}$into one set of phospholipid vesicles, and ATP into another. Fusion was studied by measuring the luminescence that results upon mixing of the intravesicular contents.

\section{Materials and Methods}

Soybean phospholipids (Sigma) was purified according to Kagawa and Racker (1971). On planar bilayer membranes, it was determined using the method of McLaughlin, Szabo \& Eisenman (1971) that there was one negative charge per 3-4 phospholipid molecules. Firefly 
luciferase over $90 \%$ pure was obtained from Sigma. Stock luciferin solution was prepared by dissolving $0.4 \mathrm{mg} / \mathrm{ml}$ luciferin (Sigma) in water adjusted to $\mathrm{pH} 8$. The $\mathrm{pH}$ was readjusted to $\mathrm{pH} 7$ prior to storage at $-10^{\circ} \mathrm{C}$.

The standard solution in all experiments was $210 \mathrm{~mm}$ sucrose, $60 \mathrm{~mm}$ HEPES, pH 7.7. Additions made to this solution are indicated in the text. Luminescence was determined in an ambient temperature, Packard liquid scintillation counter in noncoincidence mode. Gain was $13 \%$ and window settings were either $60-65$ or $50-75$. Consecutive $0.1-\mathrm{min}$ counts were taken.

Luciferase vesicles (LV) were prepared by sonication of $100 \mathrm{mg}$ phospholipid with $0.25-0.50 \mathrm{mg}$ luciferase in $1 \mathrm{ml}$ standard solution containing $2 \mathrm{mM} \mathrm{MgCl}_{2}$. Sonication was performed in a bath type sonicator for $3 \mathrm{~min}$ at room temperature under $\mathrm{N}_{2}$. The solution was then applied to either a Biogel P300 (50 mesh) or Sepharose 6B column $(26 \mathrm{~cm} \times 1.5 \mathrm{~cm})$ equilibrated with the standard solution. Standard solution was used to elute the vesicles. Fractions were assayed by addition of $30 \mu \mathrm{l}$ of a fraction to a scintillation vial with $60 \mu \mathrm{l}$ standard solution containing $2 \mathrm{mM} \mathrm{MgCl}_{2}, 10 \mathrm{pmol} \mathrm{ATP}$, and $10 \mu \mathrm{l}$ stock luciferin solution. The luminescence recorded in the scintillation counter reflected contaminating luciferase outside the vesicles. Triton X-100 (1\%) was then added to lyse the vesicles and luminescence was again determined. The increment in light emission reflected the amount of luciferase originally within the vesicles. Triton X-100 (1\%) was found to inhibit luciferase $70 \%$ both in the presence and absence of phospholipid. In quantitation of data, this inhibition was always taken into account.

Figure 1 shows that after sonication of phospholipid in the presence of luciferase, luciferase-containing vesicles could be separated with less than $1 \%$ contamination of luciferase outside the vesicles. The vesicles appeared just after the void volume of the column. These results were obtained both with Bio Gel P300 and Sepharose 6B columns. The elution pattern of Fig. 1 lacks a following fraction of free luciferase, a result which may have been caused by instability of the enzyme on the column and by the relatively slow flow rate of the Bio Gel P300 column (12-18 hr for passage of a 10-ml void volume). With faster Sepharose $6 \mathrm{~B}$ columns, free luciferase eluted 2-3 void volumes following the vesicle fraction.

ATP-containing vesicles (AV) were prepared and assayed in a similar manner. $100 \mathrm{mg}$ phospholipid was sonicated in $1 \mathrm{ml}$ of standard solution containing $0.1 \mathrm{~mm}$ ATP. The vesicles were purified from extravesicular ATP on either Bio Gel P300 or Sepharose 6B columns. Fractions were assayed by adding $30 \mu 1$ samples to a scintillation vial with $60 \mu 1$ standard solution containing $2 \mu \mathrm{g}$ luciferase, $10 \mu \mathrm{l}$ stock luciferin solution, and $2 \mathrm{mM} \mathrm{MgCl}_{2}$. Luminescence was measured to determine ATP contamination outside the vesicles. Triton $\mathrm{X}-100(1 \%)$ was then added to lyse the vesicles in order to determine the amount of intravesicular ATP. ATP-containing vesicles were obtained with $0.1-1 \%$ extravesicular ATP contamination with either type of column.

AV fractions and LV fractions were separately pooled and used in fusion experiments. Fusion was assayed in $0.2-0.4 \mathrm{ml}$ of standard solution containing various additions as indicated. Because luciferin was found to leak out of vesicles, vesicles could not be prepared containing the cofactor. Instead, since luciferin is membrane permeable, it was always present in the fusion solution $(20-40 \mu \mathrm{g} / \mathrm{ml})$. Vesicle fusion was studied by measuring the luminescence that resulted from the addition of AV and LV to the fusion medium. Usually approximately $50 \mu \mathrm{l}$ of each type of vesicle corresponding to about $0.6 \mu \mathrm{mol}$ phospholipid were added in an experiment. The vesicles were added either separately or from a premixed concentrated solution. Experiments were performed at room temperature. Lipid phosphate was determined according to Ames and Dubin (1960). Phosphate was assayed according to Rockstein and Herron (1951).

For negative stain electron microscopy, vesicles were incubated in standard solution with additions as indicated. The conditions were identical to that used in the luminescence 


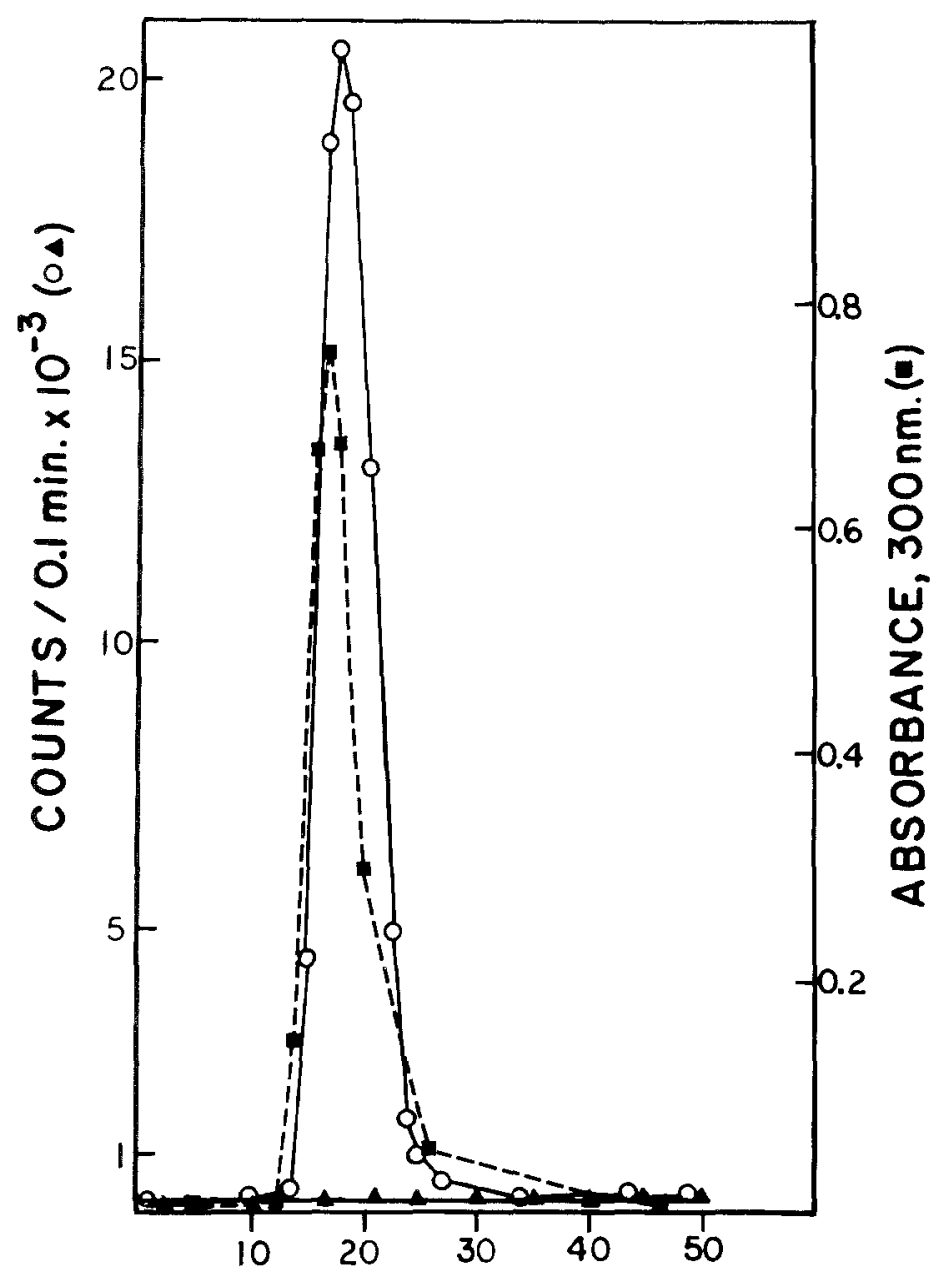

\section{FRACTION NUMBER}

Fig. 1. Purification of luciferase vesicles. Luciferase $(0.25 \mathrm{mg}$ protein) and soybean phospholipid $\left(100 \mathrm{mg}\right.$ ) were sonicated in standard solution with $10 \mathrm{mM} \mathrm{Mg}^{++}$as described in Methods. The dispersion was placed on a Bio Gel P300 column and eluted with standard solution. 0.6- $\mathrm{ml}$ fractions were collected. The flow rate was approximately $0.7 \mathrm{ml} / \mathrm{hr} .30 \mu \mathrm{l}$ from each fraction were added to a solution containing ATP, $\mathrm{Mg}^{++}$and luciferin to measure luminescence from extravesicular luciferase as described in Methods. Vesicles were then lysed with $1 \%$ Triton X-100 to determine luminescence from intravascular luciferase. To determine the elution pattern of vesicles, the absorbance at $300 \mathrm{~nm}$ was determined on 10-fold diluted aliquots. $\boldsymbol{\Delta}$, luminescence with intact vesicles; $O$, luminescence with lysed vesicles; and $\mathbf{\square}$, absorbance at $300 \mathrm{~nm}$

studies. After $1-3 \mathrm{~min}, 20 \mu \mathrm{l}$ of the reaction solution and $20 \mu \mathrm{l}$ of $3 \%$ phosphotungstic acid were mixed on carbon-coated grids. The solution was removed from the grid by absorption with filter paper. Vesicles were viewed with an AEI Corinth 275 electron microscope.

Optical density was measured at 300 or $507 \mathrm{~nm}$ in a Gilford $300 \mathrm{~N}$ spectrophotometer. 


\section{Results}

\section{Osmotic Stability of Vesicles}

The osmotic stability of vesicles was investigated by adding AV to either isotonic or hypotonic medium containing luciferase (Table 1). Light emission in isotonic medium prior to addition of Triton $X-100$ was $3.4 \%$ of light emission after addition of Triton X-100 (after complete lysis). Light emission in hypotonic medium prior to addition of Triton X-100 was $38 \%$ that after addition of Triton X-100. With the $70 \%$ inhibition of luciferase by Triton X-100 taken into account, the corrected percentage light emission compared to that after complete lysis was $1 \%$ in isotonic medium and $11 \%$ in hypotonic medium. Vesicles, therefore, became only partially leaky to ATP in hypotonic medium.

\section{Fusion Induced by $\mathrm{Ca}^{++}$and $\mathrm{Mg}^{++}$}

Because $\mathrm{Ca}^{++}$plays an important role in the fusion of intracellular secretory vesicles with the plasma membrane and is an important fusogen in artificial lipid systems, the ability of $\mathrm{Ca}^{++}$and other divalent ions to induce fusion of $\mathrm{AV}$ and $\mathrm{LV}$ was investigated. A mixture of $\mathrm{AV}$ and LV emitted light in the presence, but not in the absence of $\mathrm{Ca}^{++}$ (Figs. 2 and 3). The order of addition of vesicles and calcium influenced the kinetics of the reaction. If premixed $\mathrm{AV}$ and $\mathrm{LV}$ were added to calcium-containing solution, the light emission usually rose to a steady

Table 1. Osmotic stability of phospholipid vesicles

\begin{tabular}{|c|c|c|}
\hline & \multicolumn{2}{|c|}{ Light emission (counts $/ 0.1 \mathrm{~min}$ ) } \\
\hline & Before Triton & After Triton (1\%) \\
\hline $\begin{array}{l}\text { Isotonic medium: } 210 \mathrm{~mm} \text { sucrose, } \\
60 \mathrm{~mm} \text { NaHEPES, } 5 \mathrm{~mm} \mathrm{MgCl}_{2}, \mathrm{pH} 7.7\end{array}$ & 1,432 & 42,018 \\
\hline $\begin{array}{l}\text { Hypotonic medium: } 20 \mathrm{~mm} \text { NaHEPES, } \\
5 \mathrm{mM} \mathrm{MgCl}_{2} \text {, pH } 7.7\end{array}$ & 25,850 & 68,811 \\
\hline
\end{tabular}

ATP-containing vesicles $(0.262 \mu \mathrm{mol}$ phospholipid) were added to the above solutions which contained, in addition, $40 \mu \mathrm{g} / \mathrm{ml}$ luciferin and $2 \mu \mathrm{g}$ luciferase.

Final volume, $1.0 \mathrm{ml}$. Background of 1,800 counts $/ 0.1$ min has been subtracted. 

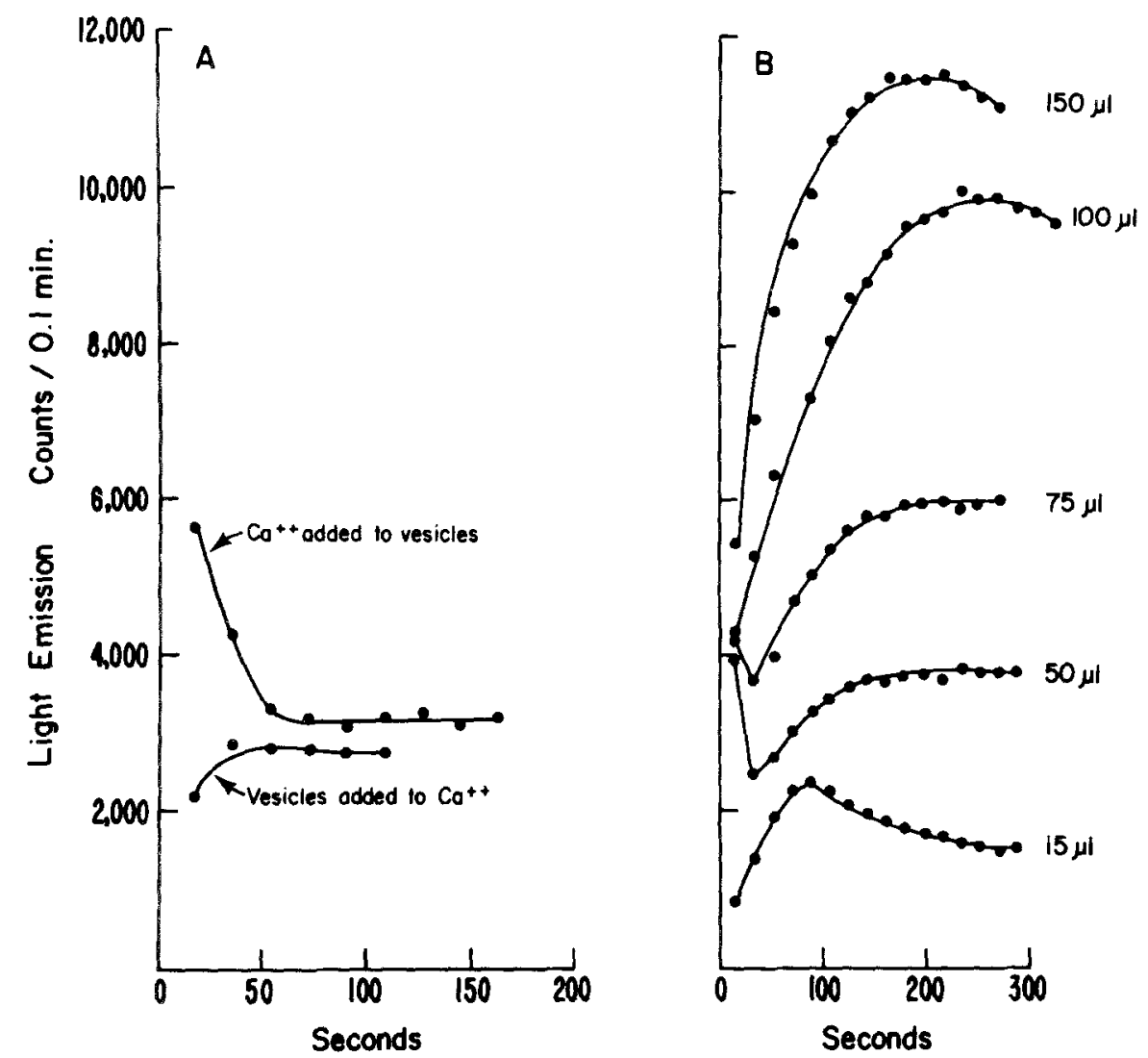

Fig. 2. Time course of calcium-induced light emission. $(A):$ Top curve: $\mathrm{CaCl}_{2}$ was added from a concentrated stock solution to LV-AV premixed (in $0.36 \mathrm{ml}$ ) in the absence of $\mathrm{Ca}^{++}$. Bottom curve: a concentrated $\mathrm{Ca}^{++}$-free mixture of $\mathrm{LV}-\mathrm{AV}$ was added to solution $(0.37 \mathrm{ml})$ already containing $\mathrm{CaCl}_{2}$. In both cases the final $\mathrm{Ca}^{++}$concentration was $10 \mathrm{mM}$, the final solution volume was $0.40 \mathrm{ml}$, and $\mathrm{AV}$ and $\mathrm{LV}$ corresponded to 0.24 and $0.20 \mu \mathrm{mol}$ phospholipid, respectively. (B): Varying amounts of a concentrated mixture of AV and $\mathrm{LV}$ were added to solution containing $10 \mathrm{mM} \mathrm{CaCl}_{2}$ (final concentration). $100 \mu \mathrm{l}$ of the vesicle mixture contained $0.68 \mu \mathrm{mol}$ phospholipid of $\mathrm{AV}$ and $0.80 \mu \mathrm{mol}$ phospholipid of LV. Final volume was $0.40 \mathrm{ml}$

constant value that persisted for 1-2 min and then slowly declined (Figs. 2A and 3). However, if calcium were added from a concentrated solution to medium containing $\mathrm{AV}$ and $\mathrm{LV}$, light emission was usually maximal at the earliest time point (approximately $15 \mathrm{sec}$ after calcium addition) and then rapidly declined to approximately the same steadystate value obtained when vesicles were added to calcium-containing medium. 

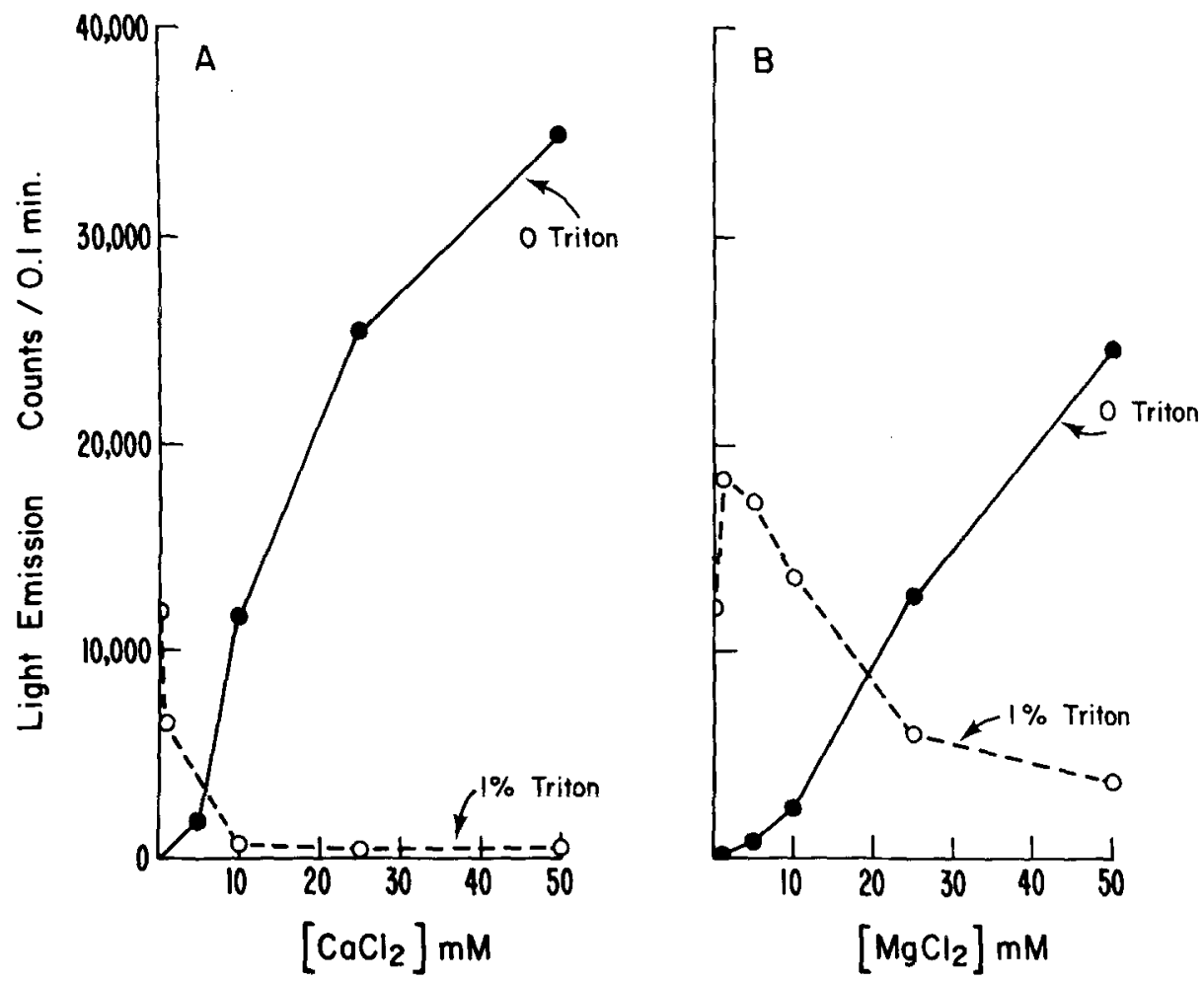

Fig. 3. The effects of varying $\mathrm{CaCl}_{2}(A)$ or $\mathrm{MgCl}_{2}(B)$ concentrations on steady-state luminescence. A concentrated divalent ion-free mixture of AV and LV $(0.52 \mu \mathrm{mol}$ phospholipid each) was added to solutions containing various concentrations of $\mathrm{Ca}^{++}$or $\mathrm{Mg}^{++}$. The final volume was $0.40 \mathrm{ml}$. After steady-state values were attained (after 2-3 min) Triton $\mathrm{X}-100(1 \%)$ was added to lyse the vesicles

The large light emission immediately following addition of $\mathrm{Ca}^{++}$ from a concentrated solution to medium containing mixed AV and LV may have resulted from a subpopulation of vesicles experiencing a shortlived high concentration of $\mathrm{Ca}^{++}$which in turn induced a locally high degree of fusion. Sometimes, however, even when vesicles were added to $\mathrm{Ca}^{++}$-containing medium, a simple rise to a steady-state level of light emission did not occur (Fig. 2B, 15, 50 and $75 \mu 1$ ). However, after 2 min a constant level of light emission was generally attained that persisted for at least one min. This steady-state level of light emission was used in tables and figures presented below.

The constant luminescence attained between 2-3 min in different $\mathrm{Ca}^{++}$concentrations is plotted in Fig. $3 \mathrm{~A}$. Calcium caused a concentration-dependent increase in light emission. $\mathrm{Mg}^{++}$caused similar effects 
but was less active than $\mathrm{Ca}^{++}$. These data suggest that $\mathrm{Ca}^{++}$and $\mathrm{Mg}^{++}$ induced fusion of $\mathrm{AV}$ and $\mathrm{LV}$ with consequent mixing of the intravesicular compartments. However, an alternative explanation is that the increased light emission resulted from reaction of vesicle contents in the medium after leakage from the vesicles. This latter possibility was intensively investigated; the results strongly suggest that leakage did not occur to a significant degree.

\section{Evidence that Divalent Ion-Induced Light Emission is Not Caused by Vesicle Lysis or Leakage from Vesicles}

Although $\mathrm{Ca}^{++}$promoted light emission in a mixture of $\mathrm{AV}$ and $\mathrm{LV}$, it is a potent inhibitor of free luciferase. This is readily seen in Fig. $3 \mathrm{~A} . \mathrm{Ca}^{++}$promoted light emission in a mixture of intact $\mathrm{AV}$ and LV, but strongly inhibited the light emission after lysis. Similarly, concentrations of $\mathrm{Mg}^{++}$greater than $2 \mathrm{~mm}$ stimulated light emission in intact vesicles, but inhibited light emission in lysed vesicles. Effects of $\mathrm{Ca}^{++}$ and $\mathrm{Mg}^{++}$similar to those obtained with Triton $\mathrm{X}$-100-lysed vesicles were obtained with free luciferase (in the absence of vesicles and Triton $\mathrm{X}-100$ ). Thus, light emission in a mixture of LV and AV in the presence of $\mathrm{Ca}^{++}$or $\mathrm{Mg}^{++}$cannot be caused by divalent ion stimulation of luciferase already present in the medium. Furthermore, it is unlikely that any leakage induced by divalent ions can account for the light emission, since $100 \%$ lysis in the presence of greater than $10 \mathrm{mM} \mathrm{Ca}^{++}$ resulted in almost no luminescence.

The possibility that light emission resulted from extravesicular ATP and luciferase was further investigated by examining the effects of EDTA.

Fig. 4. $(A)$ : The effects on luminescence of EDTA addition at various times during an incubation in the presence of $\mathrm{CaCl}_{2}$. A concentrated divalent ion-free mixture of $\mathrm{AV}$ and $\mathrm{LV}(0.66$ and $0.82 \mu \mathrm{mol}$ phospholipid of $\mathrm{AV}$ and $\mathrm{LV}$, respectively) was added to a solution containing $10 \mathrm{mM} \mathrm{CaCl}_{2}$ at zero time (arrow $V$ ) and the luminescence monitored. In three parallel experiments, EDTA (13.3 mM EDTA) was added at various times (indicated by the arrows) after vesicle addition. In one experiment $\mathrm{Ca}^{++}$and EDTA were premixed before the addition of vesicles to the solution. The concentrated EDTA solution contained sufficient $\mathrm{NaOH}$ to maintain the final solution $\mathrm{pH}$ at 7.7 after complete $\mathrm{Ca}^{++}$chelation. Note that EDTA caused a large but transient increase in luminescence. $(B)$ : The ability of a second addition of calcium to stimulate a second cycle of fusion after chelation of the original calcium in solution. Vesicles were added to $\mathrm{Ca}^{++}$-containing solution $(10 \mathrm{mM})$, and the luminescence was monitored. In 2 parallel experiments, EDTA (13.3 $\mathrm{mm})$ was added at $15 \mathrm{sec}$ to completely chelate the $\mathrm{Ca}^{++}$. The concentrated EDTA solution 


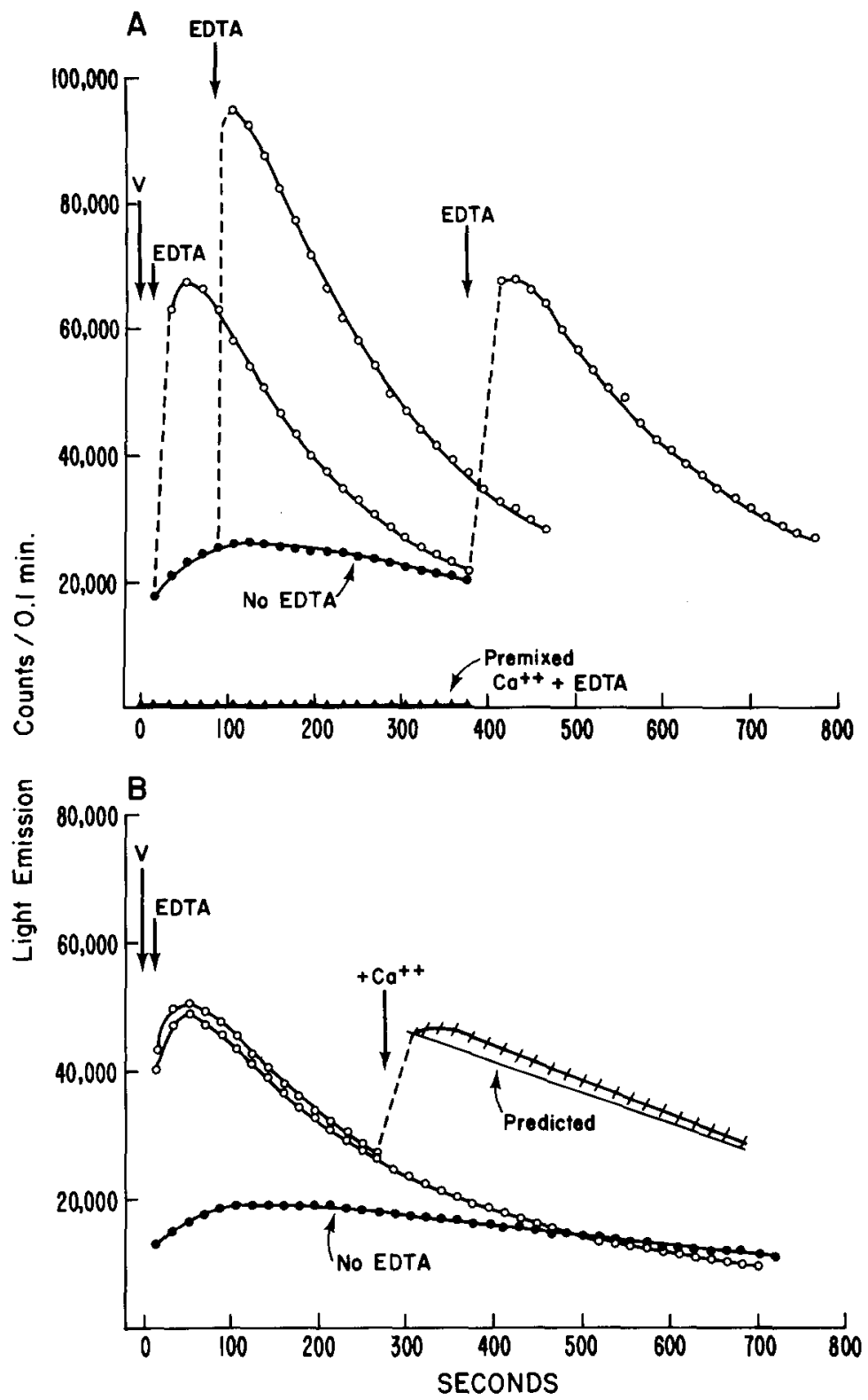

contained sufficient $\mathrm{NaOH}$ to maintain the final solution $\mathrm{pH}$ at 7.7 . In one of these experiments $\mathrm{Ca}^{++}$was subsequently added at $275 \mathrm{sec}$ to give a final free $\mathrm{Ca}^{++}$concentration of $10 \mathrm{~mm}$. In an experiment not shown, $10 \mathrm{~mm} \mathrm{Ca}^{++}$was added to a mixture of $\mathrm{AV}$ and LV which had not been previously exposed to $\mathrm{Ca}^{++}$or EDTA. The luminescence obtained in this experiment was added, beginning at $275 \mathrm{sec}$, to the falling phase of luminescence after chelation of the original calcium by EDTA. The derived line gives the predicted luminescence if the second cycle of $\mathrm{Ca}^{++}$-induced fusion is unaltered by the first cycle. $\bullet \bullet, 10 \mathrm{mM} \mathrm{Ca}^{++}$, no EDTA; $-0-0-0-0,10 \mathrm{mM} \mathrm{Ca}^{++}$chelated with $13.3 \mathrm{~mm}$ EDTA; HWt, second exposure to $10 \mathrm{~mm}$ free $\mathrm{Ca}^{++}$after an initial exposure to and subsequent chelation of $10 \mathrm{~mm} \mathrm{Ca}^{++}$; and —-, predicted luminescence. (see text) 
EDTA added in excess of divalent ions completely inhibited free luciferase and eliminated residual light emission that occurred in the presence of $\mathrm{Ca}^{++}$(data not shown). If luciferase outside the vesicles were responsible for light emission during calcium-induced fusion, then the addition of EDTA to completely chelate $\mathrm{Ca}^{++}$should stop light emission. Figure 4 demonstrates that just the opposite happened. Addition of EDTA after calcium had induced light emission in a mixture of AV and LV caused a two- to threefold increase in luminescence. This light emission must have been produced in a space which contained both ATP and luciferase but not EDTA. The experiment provides strong evidence that light emanated from fused vesicles. Note that if $\mathrm{Ca}^{++}$and EDTA were premixed, no light emission occurred. In the absence of $\mathrm{Ca}^{++}$, EDTA alone did not induce light emission (data not shown). The enhancement of light emission by EDTA, therefore, required previous exposure to $\mathrm{Ca}^{++}$; furthermore, the magnitude of the enhancement of luminescence by EDTA was directly dependent upon the magnitude of the $\mathrm{Ca}^{++}$-induced luminescence at the time of addition of EDTA (Fig. $4 A$ ). The $\mathrm{Ca}^{++}$induced light emission in the absence of EDTA reached a broad maximum or steady-state and then slowly declined with time $\left(t_{1 / 2}=640 \mathrm{sec}\right)$ (Fig. 4). After addition of EDTA to chelate $\mathrm{Ca}^{++}$, luminescence rapidly attained a maximum and then rapidly declined $\left(t_{1 / 2}=200 \mathrm{sec}\right.$ ) (Fig. 4). These results suggest that the rate of decay of luminescence in the presence of $\mathrm{Ca}^{++}$resulted from two opposing processes-decay in the intrinsic luminescence reaction in fused vesicles and continuing $\mathrm{Ca}^{++}$-induced fusion ${ }^{1}$. Chelation of $\mathrm{Ca}^{++}$prevented the continuing fusion and, thereby, increased the observed rate of decay of luminescence.

The extent of leakage of vesicle contents during fusion was investigated. The activity of free luciferase in the presence of $10 \mathrm{mM} \mathrm{Ca}^{++}$ is $2-4 \%$ of maximal activity. This activity, although small, is readily measurable and can be used to detect ATP. To study leakage during fusion induced by $10 \mathrm{mM} \mathrm{Ca}^{++}$, AV were fused with themselves (no LV present) in the presence of free luciferase and luciferin. Light emission could only result from ATP that had exited from the vesicles. Although $\mathrm{Ca}^{++}$did induce light emission, it could account for only $1 \%$ of the total possible light emission that occurred after $100 \%$ lysis by Triton $\mathrm{X}-100^{2}$. Thus, there was at most $1 \%$ leakage of ATP during incubation of $\mathrm{AV}$ with $10 \mathrm{~mm} \mathrm{Ca}^{++}$.

${ }^{1} T_{1 / 2}$ for the decay of luminescence from free luciferase is approximately $200 \mathrm{sec}$ in $50 \mathrm{~nm}$ ATP and $13 \mu \mathrm{g} / \mathrm{ml}$ luciferase and decreases with increasing ATP and luciferase concentrations.

${ }^{2}$ The $70 \%$ inhibition of luciferase by Triton X-100 is included in the estimate. 
Table 2. Determination of the amount of ATP leakage during fusion of ATP-containing vesicles

Light emission (counts/0.1 min)

Luciferase $(2 \mu \mathrm{g})+$ luciferin $(4 \mu \mathrm{g})$ 162

+ ATP-containing vesicles $(1 \mu \mathrm{mol}$ phospholipid)

$+\mathrm{CaCl}_{2}(10 \mathrm{~mm})$

+ Triton $(1 \%)$

40,439

Sequential additions as indicated were made to $90 \mu \mathrm{l}$ standard solution. The Ca-induced light emission results from ATP appearing free in solution; it accounts for $3 \%$ of the total possible counts obtained after lysis by Triton X-100. Because Triton X-100 inhibits luciferase by $70 \%$, the calcium-induced light emission represents only $1 \%$ of the total possible counts.

Although the leakage of luciferase from LV during $\mathrm{Ca}^{++}$-induced fusion was not examined, it is probable that luciferase $(100,000 \mathrm{~mol}$ wt) is released into the medium to the same or lesser degree than ATP from AV. In the above experiment (Table 2) with $2 \mu \mathrm{g}$ luciferase in the medium, 1,000 counts/0.1 min resulted upon addition of $\mathrm{Ca}^{++}$. Because the amount of luciferase in the medium in the reaction mixture was much larger than the amount (approximately $0.03 \mu \mathrm{g}$ ) which might be released by $1 \%$ leakage from $L V$ in a mixed vesicle experiment, calcium-induced leakage or lysis cannot account for the calcium-induced light emission, which was usually $10,000-20,000$ counts $/ 0.1 \mathrm{~min}$. These results, therefore, also confirm the conclusion drawn from Fig. $3 A$ that the $\mathrm{Ca}^{++}$-induced light emission was not occurring outside the vesicles.

\section{Quantitation of Extent of Fusion}

To understand some of the details of the fusion process, it was important to determine the fraction of vesicles that fuse. $100 \%$ fusion is represented by the amount of luminescence that would occur if the internal volumes of all the vesicles present in an assay coalesced into one common space. This could not be determined directly. However, by using a method described in the Appendix, the maximal light emission was extrapolated from the luminescence that resulted from lysis of a mixture of $\mathrm{AV}$ and $\mathrm{LV}$ in the presence of $\mathrm{Mg}^{++}$. Required in the calculation is an estimate of the internal volume of the vesicles. This was determined by lysing AV with Triton X-100 in the presence of luciferase (and luciferin) and determining the amount of ATP released. There was $0.9 \mu \mathrm{l}$ intravesicular volume/ $\mu \mathrm{mol}$ phospholipid (see Appendix). 
An estimate of the extent of fusion occurring in Fig. 4 (a typical experiment) is made in the Appendix. In this experiment the maximal observed luminescence was approximately 100,000 counts $/ 0.1 \mathrm{~min}$. 43,000 counts $/ 0.1$ min resulted from lysis of the same number of vesicles in $0.3 \mathrm{ml}$ solution containing $2 \mathrm{mM} \mathrm{MgCl}_{2}$. From these data one estimates that $0.1-0.2 \%$ of the vesicles had fused. Thus, only a small fraction of the total possible fusion events occurred during a cycle of fusion.

Consistent with the conclusion that only a small fraction of the vesicles fused in response to $\mathrm{Ca}^{++}$was the finding that a second cycle of fusion could be initiated by $\mathrm{Ca}^{++}$after the first cycle had been halted by EDTA (Fig. 4B). An important question is whether the second round of fusion was altered by the first round. In an experiment not shown, the luminescence was determined after $\mathrm{Ca}^{++}(10 \mathrm{~mm})$ was added to the same amount of mixed vesicles used in Fig. 4. When this luminescence was added to the rapidly falling phase (open circles) after EDTA was added to chelate calcium, the summed light emission (line without symbols) accurately predicted the luminescence from the second round of fusion. Thus, the actual increment in light emission caused by the second round of fusion was almost precisely equal to the amount of light emission from vesicles which had not been previously fused. The result strongly suggests that if $\mathrm{Ca}^{++}$is removed after $\mathrm{Ca}^{++}$-induced fusion most of the vesicles are unaltered and are once again available for fusion. This result supports the conclusion that only a very small fraction of vesicles fuse after exposure to $\mathrm{Ca}^{++}$.

Another type of experiment gave results consistent with only a small fraction of vesicles fusing. Luminescence increased when increasing concentrations of $\mathrm{Ca}^{++}$were added to the same mixture of $\mathrm{AV}$ and LV (data not shown). This suggests that in a vesicle population that had already been exposed to a substantial amount of $\mathrm{Ca}^{++}(10 \mathrm{~mm}$ or greater) there is a significant pool of unfused vesicles which can be induced to fuse by further $\mathrm{Ca}^{++}$addition.

\section{Vesicle Aggregation and the Effect of Vesicle Preincubation in $\mathrm{Ca}^{++}$-Containing Medium on Subsequent Fusion}

$\mathrm{Ca}^{++}$flocculated the lipid vesicles. The process was readily seen by eye and was measured by the absorbance at $507 \mathrm{~nm}\left(A_{507}\right)$. With a total of $5.91 \mu \mathrm{mol}$ phospholipid in $1.24 \mathrm{ml}, 10 \mathrm{mM} \mathrm{CaCl}_{2}$ caused an increase of $A_{507}$ from 0.207 to 0.500 which was completely reversed by subsequent chelation of the calcium by EDTA (13 mm). The absorb- 
Table 3. The effect of vesicle preincubation in $\mathrm{Ca}^{++}$-containing medium on fusion

Maximum light emission

(counts/0.1 min)

$\begin{array}{lr}\mathrm{AV} \text { added to } \mathrm{LV} \text { preincubated in } 10 \mathrm{mM} \mathrm{CaCl}_{2}{ }^{\mathrm{a}} & 1,675 \\ \mathrm{LV} \text { added to } \mathrm{AV} \text { preincubated in } 10 \mathrm{mM} \mathrm{CaCl}_{2}{ }^{\mathrm{b}} & 1,527 \\ \mathrm{AV} \text { and } \mathrm{LV} \text { premixed before } 10 \mathrm{mM} \mathrm{CaCl}_{2} \text { addition }^{\mathrm{c}} & 21,890\end{array}$

${ }^{\mathrm{a}} \mathrm{LV}$ was added to standard solution containing $10 \mathrm{mM} \mathrm{CaCl}_{2}$. After 2 min incubation, AV was added.

${ }^{\mathrm{b}} \mathrm{AV}$ was added to standard solution containing $10 \mathrm{mM} \mathrm{CaCl}_{2}$. After 2 min incubation, LV was added.

c AV and LV were premixed in standard solution without divalent ions, and were then added to standard solution containing $10 \mathrm{mM} \mathrm{CaCl}_{2}$.

Final volume in the experiments was $0.400 \mathrm{ml}$. The amount of AV and LV added corresponded to $1 \mu \mathrm{mol}$ phospholipid for each set of vesicles. Both LV and AV contained $2 \mathrm{mM} \mathrm{MgCl}_{2}$.

ance increase caused by $10 \mathrm{~mm} \mathrm{Ca}^{++}$was within $90 \%$ of steady state in 15-20 sec. Flocculation, therefore, is not equivalent to fusion. Fusion cannot be reversed by chelation of $\mathrm{Ca}^{++}$by EDTA whereas flocculation is reversed. Furthermore, luminescence takes at least 1-2 min to reach steady state, whereas flocculation requires less than $20 \mathrm{sec}$. These OD changes suggest that vesicles associate but do not necessarily fuse.

Vesicles (AV or $\mathrm{LV}$ ) in the presence of $\mathrm{Ca}^{++}$had a reduced capacity to fuse with added vesicles (Table 3). After LV was preincubated for 2 min in standard solution containing $10 \mathrm{mM} \mathrm{CaCl}_{2}$, the addition of AV caused a small amount of luminescence. The luminescence was less than $10 \%$ of the luminescence that resulted from addition of the same amounts of premixed (in $\mathrm{Ca}^{++}$-free medium) $\mathrm{AV}$ and $\mathrm{LV}$ to standard solution containing $10 \mathrm{mM} \mathrm{CaCl}_{2}$. Virtually identical results were obtained when LV was added to AV which had been preincubated in $\mathrm{Ca}^{++}$-containing medium. $\mathrm{Ca}^{++}$aggregates but does not necessarily fuse vesicles (see below). It is, thus, likely that unfused, aggregated vesicles are in a stable rather than a dynamic relationship with one another. Newly added vesicles do not readily exchange with previously aggregated vesicles.

\section{Relationship between $\mathrm{Ca}^{++}$-Induced Light Emission and the Amount of Vesicles}

The relationship between light emission after $2-3 \mathrm{~min}$ in $10 \mathrm{mM} \mathrm{Ca}{ }^{++}$ and the amount of premixed $\mathrm{AV}$ and $\mathrm{LV}$ is examined in Fig. 5. Light 


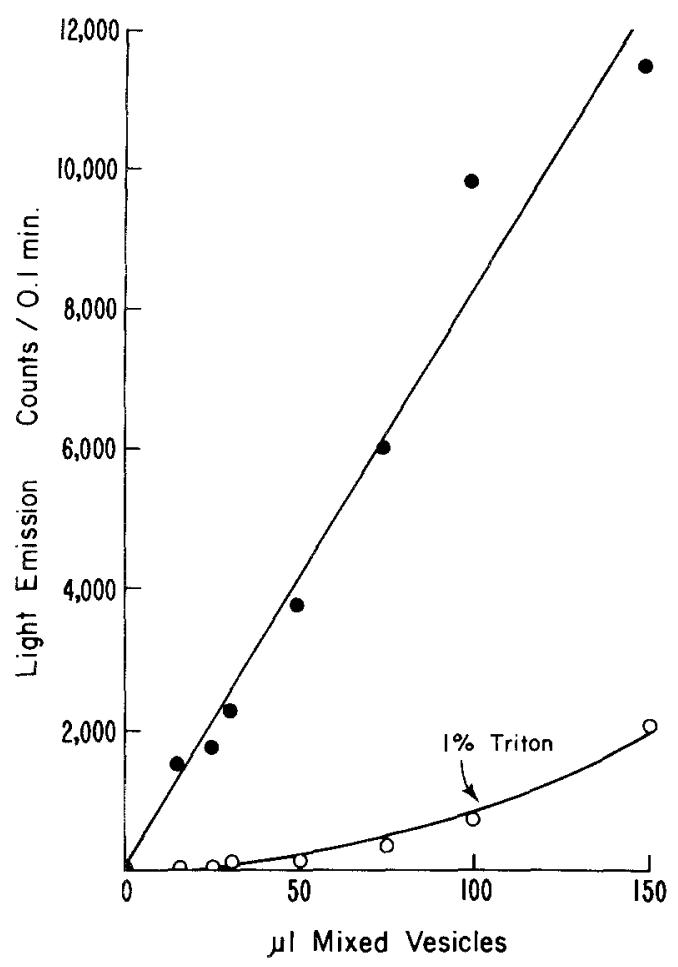

Fig. 5. The effect of increasing amounts of a mixture of AV and LV on steady-state, calcium-induced luminescence. Varying amounts of a concentrated mixture of AV and $\mathrm{LV}\left(\mathrm{Ca}^{++}\right.$-free) were added to medium containing $10 \mathrm{mM} \mathrm{CaCl}_{2}$ (closed circles); after the steady-state luminescence was attained at 2-3 min, Triton X-100 (1\%) was added to lyse the vesicle (open circles). $100 \mu \mathrm{l}$ of the concentrated mixture of vesicles contained 0.68 and $0.80 \mu$ mols phospholipid of AV and LV, respectively. Final volume was $0.40 \mathrm{ml}$. Some of the data were obtained from the time courses from Fig. $2 B$

emission increased linearly with the number of vesicles. This result suggests that a constant fraction of vesicles fused in a manner to produce light (AV fusing with LV) independent of the total number of vesicles.

The maximal amount of phospholipid in the experiment was $2.2 \mu \mathrm{mol}$, and the total amount of $\mathrm{Ca}^{++}$present was $4 \mu \mathrm{mol}$. If $\mathrm{Ca}^{++}$bound to every phospholipid molecule, then free $\mathrm{Ca}^{++}$would have been significantly reduced. However, the linearity of fusion with the amount of vesicles indicates that fusion was not limited by reduction of free $\mathrm{Ca}^{++}$ in solution and suggests that extensive $\mathrm{Ca}^{++}$binding did not occur. These results are consistent with those of Ingolia and Koshland (1978) who found that only $0.05 \mathrm{~mol} \mathrm{Ca}{ }^{++} / \mathrm{mol}$ phospholipid was associated with the soybean phospholipid vesicles in $5 \mathrm{~mm} \mathrm{CaCl}$. 

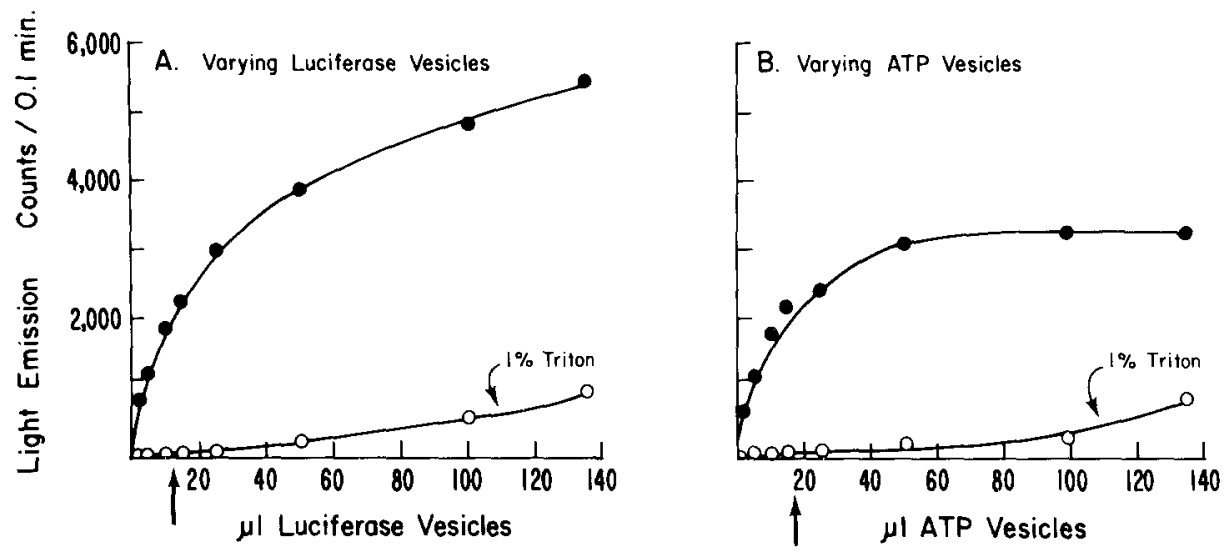

Fig. 6. The effects of separately varying $\mathrm{LV}$ or $\mathrm{AV}$ on the steady-state $\mathrm{Ca}^{++}$-induced luminescence. $(A)$ : Varying amounts of $\mathrm{LV}$ were premixed with $15 \mu 1 \mathrm{AV}$ before addition to $10 \mathrm{mM} \mathrm{CaCl}_{2}$-containing solution. After the steady-state luminescence was attained at 2-3 min, vesicles were lysed with $1 \%$ Triton X-100. $(B)$ : Varying amounts of AV were premixed with $15 \mu \mathrm{LV}$ before addition to $10 \mathrm{mM} \mathrm{CaCl}_{2}$-containing solution. Conditions were the same as in $A$. Arrows in $A$ and $B$ indicated the point where the amounts of $\mathrm{AV}$ and LV phospholipid were equal. $15 \mu \mathrm{l}$ of LV or AV contained 0.24 or $0.20 \mu \mathrm{mol}$ phospholipid, respectively. Closed and open circles correspond, respectively, to luminescence before and after lysis with $1 \%$ Triton X-100

The effect on fusion of varying the amount of one type of vesicle while the amount of the other remained constant was also investigated. As the number of one type of vesicle increases, the probability of a single vesicle of that type fusing with a like vesicle increases and the probability that it will fuse with an unlike vesicle, whose number is constant, decreases. Because only fusion between unlike vesicles results in light emission, luminescence should eventually saturate. Indeed, when either the amount of $\mathrm{LV}$ or $\mathrm{AV}$ increased while the amount of the other type of vesicle was constant, luminescence increased sublinearly or saturated (Fig. 6).

The Ability of Various Polyvalent Ions and Hypotonicity to Induce Fusion

The ability of a variety of different polyvalent ions to induce fusion was examined (Table 4). All the polyvalent ions investigated were able to induce fusion at $10 \mathrm{~mm}$ concentration. $\mathrm{Mn}^{++}$was most effective, 
Table 4. The ability of various polyvalent ions to induce fusion

\begin{tabular}{lcc}
\hline & \multicolumn{2}{c}{ Light emission (counts/0.1 min) } \\
\cline { 2 - 3 } & Before lysis & After lysis \\
\hline No polyvalent ion & 550 & - \\
$\mathrm{MnCl}_{2}$ & 57,300 & 23,300 \\
$\mathrm{LaCl}_{3}$ & $38,800^{\mathrm{a}}$ & 600 \\
$\mathrm{CaCl}_{2}$ & 21,500 & 1,750 \\
$\mathrm{SrCl}_{2}$ & 21,500 & 4,150 \\
$\mathrm{MgCl}_{2}$ & 9,750 & 33,300 \\
$\mathrm{BaCl}_{2}$ & 5,250 & 1,500 \\
\hline
\end{tabular}

Premixed AV and LV (0.655 and $0.822 \mu \mathrm{mol}$ phospholipid, respectively) were added to $0.30 \mathrm{ml}$ of solution containing $10 \mathrm{~mm}$ of the indicated polyvalent ion. Except in the presence of $\mathrm{La}^{+++}$, light emission rose to a maximum that continued for over a minute and then slowly declined. The values in the table are the maximal light emissions. Vesicles were lysed by the addition of $1 \%$ Triton X-100.

" $\mathrm{La}^{+++}$-induced light emission declined monotonically. The value in the table is the first (and maximal) value at $15 \mathrm{sec}$.

Table 5. Enhanced luminescence resulting from chelation of polyvalent ions

\begin{tabular}{ll}
\hline & $\begin{array}{l}\text { Relative luminescence } \\
\text { after polyvalent ion chelation }\end{array}$ \\
\hline $\mathrm{MnCl}_{2}$ & 2.1 \\
$\mathrm{LaCl}_{3}$ & 1.5 \\
$\mathrm{CaCl}_{2}$ & 3.2 \\
$\mathrm{SrCl}_{2}$ & 1.9 \\
$\mathrm{MgCl}_{2}$ & 1.4 \\
$\mathrm{BaCl}_{2}$ & 2.6 \\
\hline
\end{tabular}

To determine the effects of polyvalent ion chelation on luminescence, premixed AV and LV ( $1 \mu \mathrm{mol}$ phospholipid each) were added to standard solution containing $10 \mathrm{~mm}$ polyvalent ion. The effect of EDTA on the luminescence induced by polyvalent ions was determined after the polyvalent ion-induced luminescence attained steady state. For all the divalent ions the steady state corresponded to the maximal luminescence. For $\mathrm{La}^{+++}$ the steady state corresponded to luminescence which was $67 \%$ of the first and maximal value at $15 \mathrm{sec}$. EDTA concentration was $13 \mathrm{~mm}$, and the stock EDTA solution contained sufficient $\mathrm{NaOH}$ to maintain the medium $\mathrm{pH}$ at 7.7 after complete polyvalent ion chelation. The first and maximal value of luminescence after polyvalent ion chelation was used in calculating relative luminescence. The relative luminescence corresponds to the ratio of post-chelation to pre-chelation light emission. 
Table 6. Effect of hypotonicity on fusion

\begin{tabular}{ll}
\hline & $\begin{array}{l}\text { Steady-state light emission } \\
\text { (counts/0.1 min) }\end{array}$ \\
\hline Isotonic addition & \\
$\quad$ Initial fusion & 15,795 \\
$\quad$ Subsequent fusion after addition of isotonic solution & 13,991 \\
Hypotonic addition & 13,197 \\
$\quad$ Initial fusion & 18,775 \\
Subsequent fusion after addition of $\mathrm{H}_{2} \mathrm{O}$ &
\end{tabular}

ATP- and luciferase-containing vesicles were premixed $\left(1: 1\right.$ phospholipid) at $4{ }^{\circ} \mathrm{C}$. An aliquot of the mixture $(1.05 \mu \mathrm{mol}$ phospholipid) was added to $210 \mathrm{~mm}$ sucrose, $60 \mathrm{~mm}$ $\mathrm{Na}$ HEPES, and $10 \mathrm{~mm} \mathrm{CaCl}_{2}, \mathrm{pH}$ 7.7. Volume was $0.40 \mathrm{ml}$. After steady-state light emission was attained, either $0.20 \mathrm{ml}$ of 210 sucrose, $60 \mathrm{mM} \mathrm{Na}$ HEPES, pH 7.7, or $0.20 \mathrm{ml} \mathrm{H} \mathrm{H}_{2} \mathrm{O}$ was added.

$\mathrm{Ca}^{++}$was moderately effective, and $\mathrm{Ba}^{++}$and $\mathrm{Mg}^{++}$were least effective. Neither the addition of $50 \mathrm{~mm} \mathrm{NaCl}$ nor $100 \mathrm{~mm}$ sucrose induced light emission (data not shown); thus, the fusogenic effects of polyvalent ions were not caused by increases in tonicity or osmolality.

The enhanced luminescence observed after chelation of $\mathrm{Ca}^{++}$(see above) was also observed after chelation of the other polyvalent ions examined (Table 5). The largest relative stimulation resulted from chelation of $\mathrm{Ca}^{++}$and the smallest relative stimulation from chelation of $\mathrm{Mg}^{++}$.

Using an assay for fusion based upon the mixing of the membrane components of vesicles, Miller and Racker (1976) demonstrated that after $\mathrm{Ca}^{++}$-induced fusion had ceased, additional fusion could be obtained if the medium were made hypotonic. These results have been confirmed using the present assay (Table 6). After steady-state light emission was obtained with $\mathrm{Ca}^{++}$, decreasing the osmolality with $\mathrm{H}_{2} \mathrm{O}$ increased light emission (fusion) $50 \%$. Addition of isotonic solution, on the other hand, was accompanied by a $10 \%$ fall in light emission.

\section{Electron Microscopy of Vesicles}

To determine the morphological correlates of vesicle fusion, the vesicles were visualized with negative stain electron microscopy (Fig. 7). 

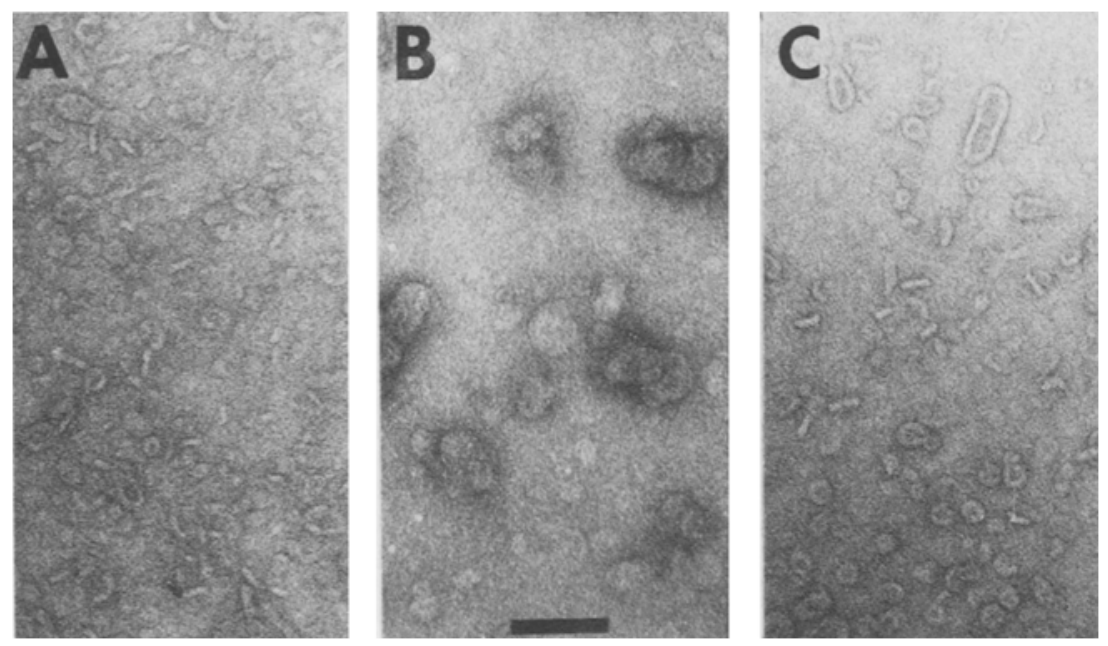

Fig. 7. Negative stain electron microscopy of vesicles in the presence and absence of $\mathrm{Ca}^{++}$. LV and AV $(0.20$ and $0.24 \mu \mathrm{mol}$ phospholipid, respectively) were mixed in $0.1 \mathrm{ml}$ divalent ion-free medium. One min after no addition $(A)$ or addition of $5 \mathrm{mM} \mathrm{CaCl}_{2}(B)$, vesicles were prepared for negative stain electron microscopy as described in Methods. In $C$, vesicles were incubated for $1 \mathrm{~min}$ in $10 \mathrm{~mm} \mathrm{CaCl}$. Calcium was then chelated with $20 \mathrm{~mm}$ EDTA. One min after addition of EDTA, the vesicles were prepared for negative stain electron microscopy. The calibration bar in B corresponds to $2,000 \AA$ in $A, B$, and $C$

Table 7. Effects of $\mathrm{Ca}^{++}$and EDTA on vesicle size measured by negative stain electron microscopy

\begin{tabular}{lcc}
\hline Additions & $n$ & Vesicle or particle diameter $^{\mathrm{a}}$ \\
\hline A) $\mathrm{None}$ & 64 & $550 \pm 35 \AA$ \\
B) $\mathrm{Ca}^{++} 5 \mathrm{mM}$ & 74 & $1,020 \pm 60 \AA^{\mathrm{b}}$ \\
C) $\mathrm{Ca}^{++} 5 \mathrm{mM}$, then EDTA $10 \mathrm{mM}$ & 75 & $515 \pm 13 \AA$ \\
D) $\mathrm{Ca}^{++} 10 \mathrm{mM}$, then EDTA $20 \mathrm{mM}$ & 74 & $592 \pm 25 \AA$ \\
\hline
\end{tabular}

Vesicles in experiments $A, B$ and $D$ were prepared for negative stain electron microscopy as described in Methods and in the legend to Fig. 7. Vesicles in $C$ were prepared as in $D$ except that $5 \mathrm{~mm} \mathrm{Ca}^{++}$and $10 \mathrm{~mm}$ EDTA were sequentially added. Measurements of vesicle size were made from extended areas of the electron micrographs, three of which are shown in Fig. 7. $n$ indicates the number of vesicles measured.

${ }^{a}$ mean \pm SEM.

${ }^{b}$ Particles appeared to be aggregates of smaller particles or vesicles (see Fig. $7 \mathrm{~B}$ ). The appearance contrasted with individual vesicles seen after either no addition, or after the sequence of $\mathrm{Ca}^{++}$followed by EDTA. $P<0.001$ compared to $A, C$ or $D$.

The vesicles were relatively heterogeneous in size with a mean diameter of $550 \AA$ (Table 7). Addition of $5 \mathrm{mM} \mathrm{CaCl}_{2}$ caused the vesicles to clump and form large particles often composed of smaller particles (Fig. $7 B$ ). 

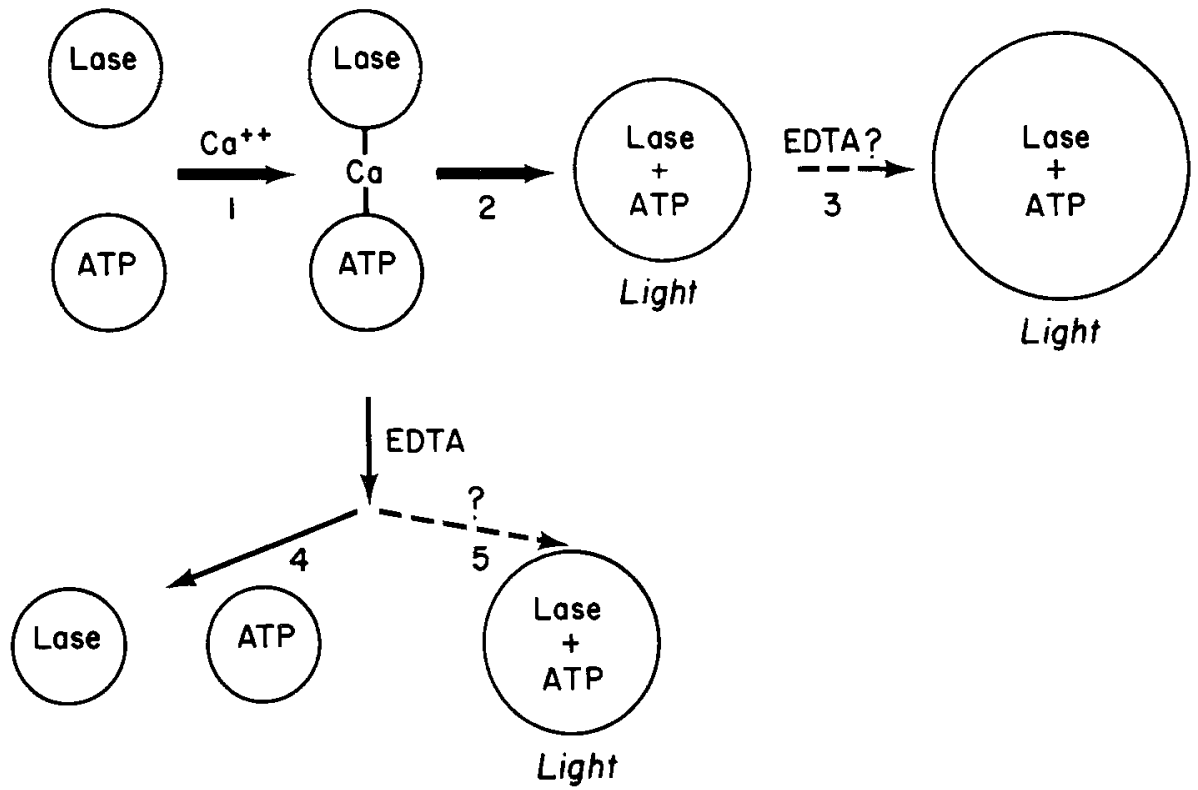

Fig. 8. Model to explain calcium-induced luminescence. Lase refers to luciferase. The circles represent vesicles in various stages of interaction or fusion. In the absence of divalent ions the vesicles do not interact. In the presence of $\mathrm{Ca}^{++}$, vesicles aggregate (arrow $l$ ) but do not necessarily fuse. A small fraction of the aggregated vesicles go on to fuse (arrow 2). The intravesicular spaces of the fused vesicles mix and luminescence results. Addition of sufficient EDTA to chelate all the $\mathrm{Ca}^{++}$disperses the aggregated vesicles (arrow 4). The increased light emission that results after $\mathrm{Ca}^{++}$chelation may be caused by an alteration in structure of the already fused vesicles (e.g., cochleate structures changing to large vesicles) (arrow 3) or by a transient second round of fusion (arow 5). (See text)

Visually, one observed the solution becoming cloudy. $10 \mathrm{mM} \mathrm{Ca}^{++}$ caused greater turbidity and larger clumps of particles (data not shown). After chelation of $\mathrm{Ca}^{++}$by EDTA, vesicles resumed their normal configuration and size (Fig. 7, Table 7), and the solution clarified. Thus, $\mathrm{Ca}^{++}$(without EDTA) aggregates vesicles without the vesicles fusing to form an extensive population of enlarged vesicles. The lack of any evident change in the vesicle size or morphology after the combination of $\mathrm{Ca}^{++}$addition and subsequent chelation supports the findings that only a small fraction of the vesicles fuse, and that the unfused vesicles have an unaltered fusion response to a second exposure to $\mathrm{Ca}^{++}$.

\section{Discussion}

\section{Divalent Ion-Induced Fusion}

A new assay has been developed and characterized to measure fusion between artificial lipid vesicles. Vesicles containing either ATP or purified 
firefly luciferase were separately prepared. Fusion between them was monitored under various conditions by measuring luminescence that resulted from the mixing of their intravesicular contents. In the absence of divalent ions, no fusion occurred. Calcium $(5-50 \mathrm{~mm})$ promoted fusion in a concentration-dependent manner without significant leakage of vesicle contents into the medium. Other polyvalent ions were also effective. Although in this study the effective $\mathrm{Ca}^{++}$concentration range is higher than generally believed to be appropriate for most biological fusion systems, it is comparable to the concentration range in other artificial membrane fusion systems (Papahadjopoulos et al., 1974; Miller \& Racker, 1976).

The results of this study suggest that $\mathrm{Ca}^{++}$-induced fusion of soybean phospholipid vesicles proceeds as depicted in Fig. 8. The essential feature of this model is that only a small fraction of the vesicles that associate in the presence of $\mathrm{Ca}^{++}$(arrow 1 ) go on to fuse (arrow 2). This conclusion is based upon 2 major findings:

1) Optical density changes and electron microscopy indicate that $\mathrm{Ca}^{++}$ induces extensive aggregation of vesicles.

2) Fusion between vesicles is an unlikely event since less than $1 \%$ of the vesicles fuse in any experiment (see Appendix). This finding is supported by the observation that after an initial round of $\mathrm{Ca}^{++}$-induced fusion, if $\mathrm{Ca}^{++}$is removed, most of the vesicles are unaltered and are once again available for fusion (Fig. 4B).

Because vesicles in the presence of $\mathrm{Ca}^{++}$have a reduced tendency to fuse (Table 3), $\mathrm{Ca}^{++}$-induced vesicle-vesicle associations are probably not readily reversible. A preset (small) fraction of the vesicles are apparently in the proper configuration to fuse.

\section{EDTA-Induced Enhancement of Luminescence}

After an initial exposure to $\mathrm{Ca}^{++}$, chelation of $\mathrm{Ca}^{++}$by EDTA induced a large increase in luminescence. The effect is not caused by reduction of the optical density due to disaggregation of the vesicles. Ingolia and Koshland (1978) examined the effects of optical density on the measured luminescence from firefly luciferase. From their data, one calculates that the optical density change in the EDTA experiment would result in only a $20 \%$ increase in luminescence. The increase in luminescence actually observed was $200-300 \%$.

Partial or complete chelation by EDTA of $\mathrm{Mg}^{++}, \mathrm{Ca}^{++}$, or the 
combination of $\mathrm{Mg}^{++}$and $\mathrm{Ca}^{++}$always inhibits and never enhances free luciferase ${ }^{3}$. In fusion experiments it is, therefore, unlikely that the enhanced luminescence after chelation of $\mathrm{Ca}^{++}$in the medium by EDTA is an artifact of the fusion assay. For example, EDTA entry into fused vesicles could only result in inhibition of luminescence, even if both $\mathrm{Ca}^{++}$(which may have entered during fusion) and $\mathrm{Mg}^{++}$are within the fused vesicles. Because in fusion experiments enhancement of luminescence occurred upon chelation of all the polyvalent ions investigated, the chelation effect is not a specific Ca-related phenomenon.

The above considerations suggest that the enhanced luminescence after chelation of $\mathrm{Ca}^{++}$and other polyvalent ions is related not to the fusion assay but to the fusion process itself. Possible mechanisms for this effect are depicted in Fig. 8. Acidic lipids form cochleate structures in the presence of $\mathrm{Ca}^{++}$which reorganize to create large vesicles upon chelation of $\mathrm{Ca}^{++}$with EDTA (Papahadjopoulos et al., 1975). Light emission induced by $\mathrm{Ca}^{++}$(Fig. 8, arrow 2) could be caused by the creation of similar cochleate structures; the enhanced light emission upon chelation of $\mathrm{Ca}^{++}$by EDTA may result from the reorganization into large vesicles (Fig. 8, arrow 3). Alternatively, as the flocculated vesicles are dispersed during chelation of $\mathrm{Ca}^{++}$by EDTA, unfused vesicles may be in a more dynamic equilibrium between themselves and residual free $\mathrm{Ca}^{++}$(before mixing and chelation are complete), thus allowing for renewed fusion. As $\mathrm{Ca}^{++}$is rapidly removed, local membrane instabilities and phase transitions may also occur that would enhance the probability of fusion (see Papahadjopoulos et al., 1977). Whatever the actual mechanism, the data suggest that not only the addition of but also the sudden removal of $\mathrm{Ca}^{++}$can induce fusion.

${ }^{3}$ The effect of $\mathrm{Ca}^{++}, \mathrm{Mg}^{++}$and EDTA were examined on the luminescence of free luciferase $(25 \mu \mathrm{g})$ in the presence of $10 \mathrm{pmol}$ ATP. The conditions were identical to those of the fusion assay. Where appropriate, EDTA contained $\mathrm{NaOH}$ to prevent alteration of the medium $\mathrm{pH}$ upon chelation of ions. In the absence of added divalent ions there is significant luciferase activity which is inhibited approximately $99 \%$ by EDTA. In $\mathrm{Mg}^{++}$ or $\mathrm{Ca}^{++}$-containing solutions, EDTA in excess of divalent ion inhibited luciferase by over $99 \%$. In the presence of $2 \mathrm{mM} \mathrm{Mg}^{++}, 1 \mathrm{~mm}$ EDTA inhibited luciferase $80 \%$. In a separate experiment, addition of $10 \mathrm{mM} \mathrm{Ca}^{++}$to luciferase (no $\mathrm{Mg}^{++}$present) reduced activity by $95 \%$. Further addition of $5 \mathrm{~mm}$ EDTA inhibited luminescence $97 \%$ compared to luminescence in $10 \mathrm{mM} \mathrm{Ca}^{++}$alone. Since $\mathrm{Ca}^{++}$inhibits luciferase, the inhibition by EDTA in this experiment suggests that the inhibition is not directly caused by chelation of $\mathrm{Ca}^{++}$. In another experiment, addition of $10 \mathrm{mM} \mathrm{Ca}^{++}$to $2 \mathrm{mM} \mathrm{Mg}^{++}$inhibited activity $97 \%$. Further addition of $5 \mathrm{~mm}$ EDTA inhibited activity $90 \%$ compared to activity in the presence of $2 \mathrm{mM} \mathrm{Mg}{ }^{++}$and $10 \mathrm{mM} \mathrm{Ca}^{++}$. It is evident that under all conditions, addition of EDTA inhibits luciferase. 
After $\mathrm{Ca}^{++}$chelation, the vesicles fuse in response to added $\mathrm{Ca}^{++}$ in an almost identical manner to vesicles that had not previously been incubated with $\mathrm{Ca}^{++}$. Hence, most of the vesicle associations induced by $\mathrm{Ca}^{++}$are reversed by calcium chelation except for the small fraction of associations that have resulted in fusion.

\section{Stability of Vesicles in Hypotonic Medium}

Phospholipid vesicles were relatively stable during incubation in hypotonic medium. In the presence of a 0.3 osmolal gradient across the vesicle membranes which corresponded to a pressure difference of $7 \mathrm{~atm}$, $90 \%$ of the ATP was retained in AV (Table 1). Planar bilayer membranes cannot withstand this large a pressure difference. However, because of the small radius of curvature, lipid vesicles $500 \AA$ in diameter in the absence of an osmolality difference across the membrane have a 16-80 atm pressure difference across the membrane ${ }^{4}$. The increment in pressure caused by the imposed osmolality difference, therefore, may be a relatively small fraction of the total pressure gradient and may not add a significant instability to the membranes. It is possible that only the larger vesicles became leaky. The effect of the small radius of curvature to increase osmotic stability may also explain the osmotic stability of sympathetic nerve storage vesicles which have a diameter of approximately $750 \AA$ (White, 1976; R.W. Holz, personal observations).

\section{Relationship to Previous Work}

While the present work was in progress, Ingolia and Koshland (1978) described an assay for soybean phospholipid vesicle fusion also based upon the luminescence of firefly luciferase. The preparation was different than that used in the present study. Crude firefly luciferase rather than purified firefly luciferase was used, and vesicles were prepared by cholate dialysis rather than by sonication. It was not indicated whether exogenous

${ }^{4}$ From Laplace's Law, the pressure difference across a lipid bilayer vesicle equals $4 \gamma / \mathrm{R}$ where $\gamma$ equals the surface tension between water/hydrocarbon interfaces and $R$ is the radius of curvature. $\gamma$ for most phospholipids equals 10-50 dyne/cm (Stein, 1967). One calculates for a vesicle with a radius of $250 \AA$, that the pressure difference is $16-80 \mathrm{~atm}$ $\left(1.0 \times 10^{6} \mathrm{dyne} / \mathrm{cm}^{3}=1 \mathrm{~atm}\right)$. 
luciferin was used in their preparation. They found, as we did, that only a small fraction of the total possible light emission resulted from $\mathrm{Ca}^{++}$-induced fusion. Because in negative stain electron microscopy they observed that $5 \mathrm{mM} \mathrm{Ca}^{++}$doubled the mean particle diameter, they concluded that virtually all the vesicles fuse, but that most of the fusion events are leaky. Our results are similar. However, because of more extensive investigation, we have concluded that the particle size increase due to $\mathrm{Ca}^{++}$is caused mainly by aggregation of vesicles and not by fusion and that there is little leakage of vesicle contents. Our conclusions are based upon both electron microscopy and fusion assay experiments. In negative stain electron microscopy, we found obvious subunit structure of the enlarged particles in $5 \mathrm{~mm} \mathrm{Ca}{ }^{++}$, which suggested that the size increase resulted from aggregation rather than fusion. Furthermore, the increase in particle size was reversed by chelation of $\mathrm{Ca}^{++}$by EDTA, an effect that is inconsistent with $\mathrm{Ca}^{++}$having induced massive fusion. Ingolia and Koshland (1978) did not investigate the effects of removing free $\mathrm{Ca}^{++}$on the $\mathrm{Ca}^{++}$-induced morphological changes. We also directly demonstrated that less than $1 \%$ leakage of intravesicular contents occurs after vesicles (AV) are placed in $\mathrm{Ca}^{++}$-containing medium. We also found that a second exposure to $\mathrm{Ca}^{++}$, after a first exposure and subsequent chelation by EDTA, resulted in light emission equivalent to that in vesicles that had not previously been incubated with $\mathrm{Ca}^{++}$. We have, therefore, concluded that fusion is a relatively rare event, and that most of the $\mathrm{Ca}^{++}$-induced vesicle-vesicle associations that occur over a time span of several minutes are reversed by chelation of $\mathrm{Ca}^{++}$. These effects are not associated with extensive leakage of vesicle contents.

In other studies, extensive enlargement of vesicle size has been observed after chelation of $\mathrm{Ca}^{++}$following $\mathrm{Ca}^{++}$-induced fusion (Papahadjopoulos et al., 1974; Miller \& Racker, 1976). In these studies, vesicles were made from different lipids and vesicles were prepared for electron microscopy after a much longer incubation in $\mathrm{Ca}^{++}$(at least $1 \mathrm{hr}$ ) than in the present studies (1-2 min). Both these differences may have enhanced the morphological effects of $\mathrm{Ca}^{++}$. In the present study, vesicles were prepared for electron microscopy during the time period when fusion was maximally occurring in order to correlate morphological changes with luminescence.

We thank Dr. Robert Gray for the electron microscopy. The research was supported by a University of Michigan Rackham Faculty Research Grant, a University of Michigan Phoenix-Memorial Grant, A PMA Foundation Research Starter Grant, and a Michigan Heart Grant. Carol A. Stratford was supported by NIH Training Grant GM 00198-19. 


\section{Appendix}

\section{Estimation of the Fraction of Vesicles that Fuse}

To calculate the fraction of vesicles fusing in an experiment, the luminescence that would result from complete and total mixing of the intravesicular spaces of $\mathrm{LV}$ and $\mathrm{AV}$ must be determined. Additional terms that must be estimated are the intravesicular volume and a correction factor to account for fusion events that do not produce light (e.g., AV-AV and LV-LV fusions).

1. Determination of intravesicular volume. To determine the intravesicular volume, the amount of ATP released from a sample of AV after lysis by Triton X-100 was measured. Vesicles were added to $100 \mu 1$ standard solution containing luciferase $(2 \mu \mathrm{g})$, luciferin $(3 \mu \mathrm{g})$, and $2 \mathrm{~mm}$ $\mathrm{MgCl}_{2}$. Triton $\mathrm{X}-100(1 \%)$ was added to lyse the vesicles and the luminescence measured. 10 pmol ATP were then added to calibrate the luminescence. From the concentration of ATP within the vesicles (the ATP concentration in which the vesicles were originally sonicated), the intravesicular volume is readily calculated. In two different preparations the values were 0.76 and $1.1 \mu 1 / \mu \mathrm{mol}$ phospholipid. The average was $0.9 \mu \mathrm{l} /$ $\mu$ mol phospholipid. This value is similar to $0.7 \mu 1 / \mu \mathrm{mol}$ phospholipid obtained by Ingolia and Koshland (1978) who used a different method. LV were assumed to have the same volume per $\mu$ mol of phospholipid.

2. Determination of the luminescence that would result from $100 \%$ fusion. In a fusion experiment $1-2 \mu 1$ intravesicular volume $(1-2 \mu \mathrm{mol}$ phospholipid) is added to $0.3-0.4 \mathrm{ml}$ buffer. When a mixture of $\mathrm{AV}$ and $\mathrm{LV}$ are lysed by Triton $\mathrm{X}-100$ in the presence of $\mathrm{Mg}^{++}$, light emission results from the mixing of the released ATP and luciferase in the total volume. This luminescence can be used to estimate the luminescence that would have occurred if the intravesicular contents were directly mixed without dilution, provided that the effects on luminescence of dilution of a constant amount of ATP and luciferase are known. Dilution effects were investigated (Fig. 9). $1 \mu \mathrm{l}$ of $0.1 \mathrm{~mm} \mathrm{ATP} \mathrm{solution} \mathrm{in} \mathrm{which}$ vesicles are sonicated to prepare $A V$ and $1 \mu \mathrm{l}$ of luciferase-containing solution $(0.56 \mathrm{mg} / \mathrm{ml})$ in which vesicles are sonicated to prepare $\mathrm{LV}$ were mixed in different volumes of solution containing $\mathrm{Mg}^{++}$and luciferin, and light emission was monitored. The most concentrated mixture that could be monitored was a $1: 4$ dilution ( 0.2 relative concentration). In a $\log -\log$ plot a straight line could be extrapolated to determine the luminescence of an undiluted mixture of $1 \mu \mathrm{l}$ each of ATP and luciferase 


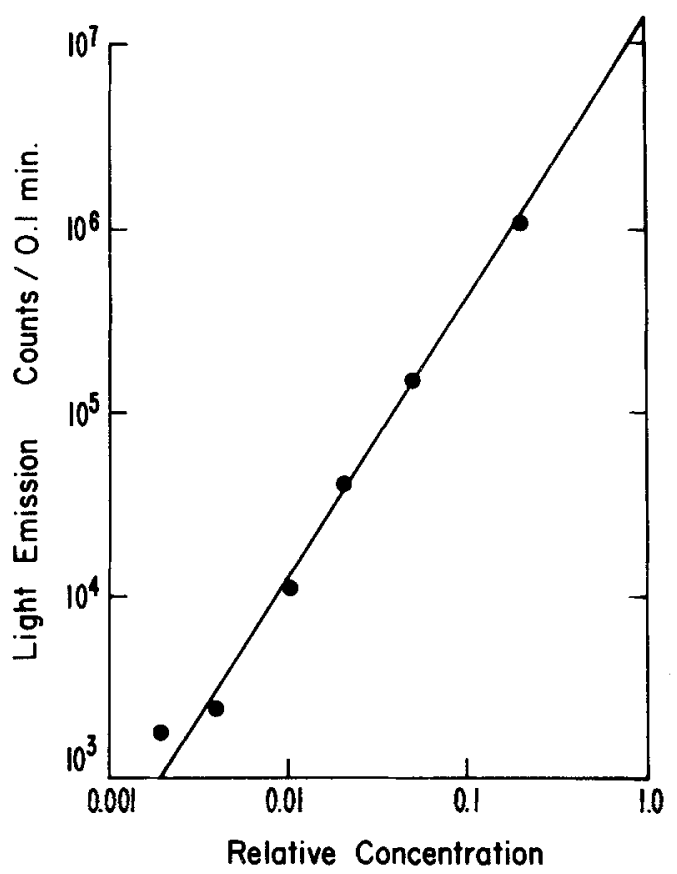

Fig. 9. The effects on luminescence of dilution of a constant amount of luciferase and ATP. ATP and luciferase solutions were prepared in a similar manner to those used in preparing AV and LV (see Methods), except that no vesicles were present and solutions were not sonicated. The luciferase-containing solution contained $0.56 \mathrm{mg}$ protein $/ \mathrm{ml}$. The ATP-containing solution contained $0.1 \mathrm{mM} \mathrm{ATP.} 1 \mu \mathrm{l}$ of each solution or the equivalent were mixed in various volumes of standard solution containing $\mathrm{MgCl}_{2}$ and luciferin, final concentrations $2 \mathrm{~mm}$ and $20 \mu \mathrm{g} / \mathrm{ml}$, respectively. Points were determined in duplicate. Initial luminescence is graphed vs. relative concentration in a log-log plot. Relative concentration equal to 1.0 corresponds to the mixing of $1 \mu$ of ATP-containing solution with $1 \mu 1$ of luciferase-containing solution. The slope of the line indicates that luminescence increases proportionally to the relative concentration raised to the 1.6 power

solutions. The slope of the line is 1.6. Thus, after lysis, if the vesicle contents are diluted $D$-fold, the luminescence expected if the contents were mixed without dilution would be $D^{1.6}$ times the luminescence after lysis (with the inhibition of luciferase by Triton X-100 taken into account). For example, in an experiment a mixture of AV and LV corresponding to a total intravesicular volume of $1.5 \mu \mathrm{l}$ was added to $0.3 \mathrm{ml}$ of solution containing $2 \mathrm{mM} \mathrm{MgCl}_{2}$. After lysis with $1 \%$ Triton X-100, the luminescence was 43,000 counts $/ 0.1$ min. Because $1 \%$ Triton X-100 inhibits luciferase by $70 \%$, the luminescence corrected for Triton inhibition was $43,000 / 0.3=143,000$ counts $/ 0.1 \mathrm{~min}$. The ATP and luciferase had been diluted 200 -fold by release into the medium. 200 raised to 
the 1.6 power is 4,805 . Therefore, the luminescence that would have been expected if complete mixing of the intravesicular contents had occurred without dilution was $143,000 \times 4,805=6.9 \times 10^{8}$ counts $/ 0.1 \mathrm{~min}$. These vesicles were used in the EDTA experiment shown in Fig. 4 where luminescence reached a maximum of 100,000 counts $/ 0.1 \mathrm{~min}$ or $0.015 \%$ of the estimated maximal light emission.

3. Correction factor to account for nonlight emitting fusion events. Fusion events between AV alone and LV alone will not result in light emission. Therefore, the observed luminescence represents only a fraction of the total fusion that is occurring. If fusion among vesicles is random, the statistics of this process can be approximated by a hypergeometric distribution (Hodges \& Lehmann, 1964). Consider, for example, the situation in which 3 vesicles fuse. The probability that light would be emitted would be given by

$$
\frac{\left(\begin{array}{c}
n_{\mathrm{L}} \\
1
\end{array}\right)\left(\begin{array}{c}
n_{\mathrm{A}} \\
2
\end{array}\right)}{\left(\begin{array}{c}
n_{T} \\
3
\end{array}\right)}+\frac{\left(\begin{array}{c}
n_{\mathrm{L}} \\
2
\end{array}\right)\left(\begin{array}{c}
n_{\mathrm{A}} \\
1
\end{array}\right)}{\left(\begin{array}{c}
n_{T} \\
3
\end{array}\right)}+\frac{\left(\begin{array}{c}
n_{\mathrm{L}} \\
1
\end{array}\right)\left(\begin{array}{c}
n_{\mathrm{A}} \\
1
\end{array}\right)\left(\begin{array}{c}
n_{0} \\
1
\end{array}\right)}{\left(\begin{array}{c}
n_{T} \\
3
\end{array}\right)}
$$

where $n_{\mathrm{L}}=$ number of luciferase containing vesicles, $n_{\mathrm{A}}=$ number of ATP containing vesicles, $n_{0}=$ number of vesicles containing neither luciferase nor ATP, $n_{T}=$ total number of vesicles, and $\left(\begin{array}{l}R \\ S\end{array}\right)$ refers to the number of combinations of $R$ number of vesicles taken $S$ at a time.

It can be shown from the hypergeometric distribution that for 2and 3-vesicle fusion, the fractions of fusion events that would involve at least one vesicle containing luciferase molecule and one vesicle containing ATP are:

$$
\begin{array}{ll}
2 f_{\mathrm{L}} f_{\mathrm{A}} & 2 \text { vesicle fusion } \\
3 f_{\mathrm{L}} f_{\mathrm{A}}\left(1+2 f_{0}\right) & 3 \text { vesicle fusion }
\end{array}
$$

where $f_{\mathrm{L}}$ and $f_{\mathrm{A}}$ are the fractions of the total number of vesicles which contain at least one luciferase molecule or ATP molecule, respectively. $f_{0}$ is the fraction of the total number of vesicles that contains neither luciferase nor ATP molecules. From electron microscopy, the average diameter of the vesicles was $550 \AA$. Usually luciferase vesicles contained $0.3 \mathrm{mg} / \mathrm{ml}$ purified luciferase. The mol wt of luciferase is approximately 100,000 . From these numbers it can be calculated that only 1 in 5 vesicles 
contains luciferase. On the other hand, each ATP-containing vesicle will contain on the average 60 ATP molecules (intravesicular concentration is $0.1 \mathrm{~mm}$ ). In most fusion experiments $\mathrm{AV}$ and $\mathrm{LV}$ are mixed in equal phospholipid ratios so that $f_{\mathrm{L}}=0.1, f_{\mathrm{A}}=0.5, f_{0}=0.4$. The fraction of fusion reactions that would result in light emission would be 0.1 and 0.2 for reactions in which 2 and 3 vesicles fuse, respectively. In general, if more vesicles fuse in a fusion reaction, then the probability of light emission resulting from a fusion reaction increases. To obtain the fraction of maximal fusion occurring, the fraction of maximal luminescence should be multiplied by at most a factor of 10 , which assumes fusion is a 2 -vesicle event. If more than 2 vesicles fuse together, the factor is smaller. In the experiment shown in Fig. 4, where the luminescence was $0.015 \%$ of maximal luminescence, the actual fusion is at most $0.15 \%$ of maximal fusion.

\section{References}

Ahkong, Q.F., Cramp, F.C., Fisher, D., Howell, J.I., Tampion, W., Verrinder, M., Lucy, J.A. 1973. Chemically-induced and thermally-induced cell fusion: Lipid-lipid interactions. Nature New Biol. 242:215

Ames, B.N., Dubin, D.T. 1960. The role of polyamines in the neutralization of bacteriophage deoxyriboneucleic acid. J. Biol. Chem. 235:769

Breisblatt, W., Ohki, S. 1976. Fusion in phospholipid spherical membranes. II. Effect of cholesterol, divalent ions and pH. J. Membrane Biol. 29:127

Deluca, M., McElroy, W.D. 1974. Kinetics of the firefly luciferase catalyzed reactions. Biochemistry 13:921

Dunham, P., Barbiarz, P., Israel, A., Zerial, A., Weissman, G. 1977. Membrane fusion: Studies with a calcium-sensitive dye arsenazo III in liposomes. Proc. Nat. Acad. Sci. USA 74:1580

Gratzl, M., Dahl, G. 1978. Fusion of secretory vesicles isolated from rat liver. J. Membrane Biol. 40:343

Hodges, J.L., Lehmann, E.L. 1964. Basic Concepts of Probability and Statistics. pp. 154-157. Holden-Day, San Francisco

Ingolia, T.D., Koshland, D.E. 1978. The role of calcium in fusion of artificial vesicles. J. Biol. Chem. 253:3821

Kagawa, Y., Racker, E. 1971. Partial resolution of the enzymes catalyzing oxidative phosphorylation. XXV. Reconstitution of vesicles catalyzing ${ }^{32} \mathrm{P}$-adenosine triphosphate exchange. J. Biol. Chem. 246:5477

Kimmich, G.A., Randles, J., Brand, J.S. 1975. Assay of picomole amounts of ATP, ADP, and AMP using the luciferase enzyme system. Anal. Biochem. 69:187

Lawson, D., Raff, M.C., Gomperts, B., Fewtrell, C., Gilula, N.B. 1977. Molecular events during membrane fusion, a study of exocytosis in rat peritoneal mast cells. J. Cell. Biol. $72: 242$

Martin, F.J., MacDonald, R.C. 1976. Lipid vesicle-cell interactions. II. Induction of cell fusion. J. Cell. Biol. 70:494 
McLaughlin, S.G.A., Szabo, G., Eisenman, G. 1971. Divalent ions and the surface potential of charged phospholipid membranes. J. Gen. Physiol. 58:667

Miller, C., Arvan, P., Telford, J.N., Racker, E. 1976. $\mathrm{Ca}^{++}$-induced fusion of proteoliposomes: Dependence on transmembrane osmotic gradient. J. Membrane Biol. 30:271

Miller, C., Racker, E. 1976. Fusion of phospholipid vesicles reconstituted with cytochrome oxidase and mitochondrial hydrophobic protein. J. Membrane Biol. 26:319

Papahadjopoulos, D., Poste, G., Schaeffer, G.E., Vail, W. 1974. Membrane fusion and molecular segregation in phospholipid vesicles. Biochim. Biophys. Acta 352:10

Papahadjopoulos, D., Vail, W.J., Jacobson, K., Poste, G. 1975. Cochleate lipid cylinders: Formation by fusion of unilamellar lipid vesicles. Biochim. Biophys. Acta 394:483

Paphadjopoulos, D., Vail, W.J., Newton, C., Nir, S., Jacobson, K., Poste, G., Lazo, R. 1977. Studies on membrane fusion: III. The role of calcium-induced phase changes. Biochim. Biophys. Acta 465:579

Rockstein, M., Herron, P.W. 1951. Colorimetric determination of inorganic phosphate in microgram quantities. Anal. Chem. 23:1500

Stanley, P.E., Williams, S.G. 1969. Use of the liquid scintillation spectrometer for determining adenosine triphosphate by the luciferase enzyme. Anal. Biochem. 29:381

Stein, W.D. 1967. The Movement of Molecules across Cell Membranes. p. 32. Academic Press, New York

White, T.D. 1976. Evidence that rapid binding of newly accumulated noradrenaline within synaptosomes involves synaptic vesicles. Brain Res. 108:87 